\title{
Prevalence of dementia in an elderly rural population: effects of age, sex, and education
}

\author{
M Prencipe, A R Casini, C Ferretti, M T Lattanzio, M Fiorelli, F Culasso
}

\begin{abstract}
Objectives-To estimate the prevalence of dementia in an elderly rural population and to determine the effects of age, sex, and education.

Methods-To obtain prevalence estimates of both cognitive impairment and dementia a door to door two phase population survey was carried out in three rural villages in central Italy. Of 1147 inhabitants older than $64,968(84.4 \%)$ completed the protocol.

Results-The prevalence rates (cases per 100 population over 64 ) were $8 \cdot 0$ for dementia and $27 \cdot 3$ for cognitive impairment. The prevalence rate for dementia did not differ between men and women (7.9 $v 8.2$ ), but increased with age (from $1 \cdot 1$ at age $65-69$ to $34 \cdot 8$ at age $90-96$ ). Subjects with less than three years of schooling had a significantly higher prevalence of dementia (14.6; $95 \%$ confidence interval $(95 \% \mathrm{CI}) 10 \cdot 2-19 \cdot 1)$ than subjects with three or more years of

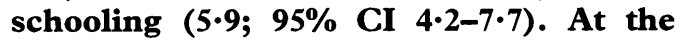
multivariate logistic analysis, the risk related with a low level of education was still present after adjustment for age and sex $\quad($ OR $=2 \cdot 0 ; \quad 95 \%$ CI $1 \cdot 2-3 \cdot 3)$. Alzheimer's disease was diagnosed in 64\% of the 78 demented patients, vascular dementia in $27 \%$, and other dementing diseases in $9 \%$.

Conclusions-In both Alzheimer and vascular dementia subtypes, the prevalence rates did not differ between men and women, but increased with age and were higher in subjects with a low level of education.
\end{abstract}

\section{$(\mathcal{F}$ Neurol Neurosurg Psychiatry 1996;60:628-633)}

Keywords: dementia; education; rural population

The progressive aging of the population is associated with a steady increase in all age related diseases. Among these, dementing disorders cause great concern because of their peculiar disabling effects. Despite the interest in this condition, prevalence estimates show pronounced variations due to differences in case ascertainment procedures, diagnostic criteria, age distribution, and the rural or urban location of the populations studied. ${ }^{1-8}$ Furthermore, education and occupation may influence the cognitive performance and increase the risk of dementia. ${ }^{-13}$
The present study analyses baseline data obtained in a door to door prospective population survey on cognitive impairment and functional disability of people older than 64 (AQUILA study). Here we report the effects of age, gender, and education on the prevalence of dementia.

\section{Methods}

We selected three rural villages (Poggio Picenze, Scoppito, Tornimparte) near the town of L'Aquila (central Italy) because of their population size (a total of 1147 people over 64 ), population stability (only $0.2 \%$ of people over 59 had moved elsewhere in the five years preceding the prevalence day), and the active collaboration offered by family doctors.

The study was conducted on all persons over 64 residing in the selected villages on the prevalence day (1 March 1992). The ascertainment of cases was made between March 1992 and February 1993 by means of a door to door two phase design. Four lay interviewers (MLB, MC, CG, and GM) and four doctors (ARC, CF, MTL, and PC) conducted the first (screening interview) and the second phases (clinical evaluation) respectively. Before the screening interview all subjects, or their relatives when appropriate, were asked for their informed consent.

\section{SCREENING INTERVIEW}

The screening interview consisted of a semistructured questionnaire (questions on education, occupation, daily life activities, previous diseases, hospital admissions, and drugs being taken) followed by the mini mental state examination (MMSE) ${ }^{14}$ or-for people with less than three years of schooling or with physical impairments hampering the MMSE evaluation-the mental status questionnaire (MSQ). ${ }^{15}$ The four lay interviewers were trained for three months, and their interrater reliability was tested by kappa statistics. ${ }^{16}$ Interrater agreement, measured in 24 subjects (positive versus negative screening test), was almost perfect $^{17}$ for both MMSE $(k=0 \cdot 88)$ and MSQ $(k=0.82)$.

Subjects with scores lower than 28 on the MMSE or lower than 10 on the MSQ were submitted to the second phase of the study. They were asked to undergo an ECG and routine blood tests, unless they had already had them in the previous six months.

Information about the physical and cognitive state of people that refused or died before the interview was obtained from relatives and 
family doctors by a scheduled questionnaire. One of the investigators (ARC) traced by phone and, whenever possible, interviewed people living in other towns.

\section{CLINICAL EVALUATION}

All subjects were examined in their place of residence within two weeks (mean seven days) of the screening interview. The examination required about two hours (range one to three hours) and included: (1) a semistructured interview of the subject's medical history integrated with the examination of all previous clinical records; (2) a close informant interview to obtain information on the medical history, current and past cognitive performances, and social and daily living activities of the subjects; the informants over 64 were judged eligible only if they had MMSE or MSQ scores above the pre-established cut off points; (3) a standard physical and neurological examination; (4) a psychiatric evaluation including the Hamilton depression rating scale (HDRS) ${ }^{18}$; (5) a structured questionnaire designed to evaluate the six categories of the clinical dementia rating (CDR) scale ${ }^{19}$ by asking questions on memory (facts of general knowledge and past and recent personal history), orientation (temporal and spatial), judgement and problem solving (similarities, differences, proverbs, ability to handle finances, and solving daily life problems), community affairs (job, shopping, and social activities), home and hobby activities (housekeeping, food preparation, responsibility for own medication, life at home, and hobbies), personal care (items on dressing, feeding, toileting, and continence taken from the disability rating scale of Broe et $a l^{20}$ ); questions on judgement and problem solving, community affairs, home and hobby activities were appropriate to age, occupation and education of the study population; (6) a standardised evaluation of the following cognitive functions: language (10 items on comprehension of verbal commands and sentences, followed by the first 15 items of the Boston naming test ${ }^{21}$ with a cut off point of 13/14), ideative praxis (five tasks of pantomime), ideomotor praxis (De Renzi test with cut off points adjusted for age and education of the Italian population ${ }^{22}$ ), and visuospatial and constructional abilities (clock drawing test $\mathrm{t}^{23}$ with a cut off point of $6 / 7$ ).

\section{DIAGNOSTIC PROCEDURES}

In the presence of an acute medical illness that could cause disturbances of attention or consciousness the evaluation was suspended and postponed until at least four weeks after recovery. Subjects affected by severe aphasia (unable to understand verbal commands or sentences), by severe depression (HDRS scores over 15), or by other psychiatric diseases hampering a reliable cognitive assessment were judged cognitively unclassifiable. The other subjects were diagnosed on the basis of combined data (person plus informant) using a two step procedure.

At the end of the first step we divided the subjects into three diagnostic categories: nor- mal, cognitively impaired, and demented. We considered as normal the subjects who had normal scores in all six categories of the CDR and in all the cognitive tests. The diagnosis of dementia was made only in subjects with duration of cognitive decline of at least six months, according to the DSM-III criteria. $^{24}$ The global CDR was graded in five levels of impairment ${ }^{25}$ : none (CDR 0), questionable (CDR 0.5), mild (CDR 1), moderate (CDR 2 ), and severe (CDR 3). We judged as demented only those subjects with a global CDR score higher than $0 \cdot 5$. Moreover, when physical handicaps or other factors hampered the evaluation of two or more functional categories (community affairs, home and hobby activities, and personal care) subjects were classified as demented only if all the remaining categories rated 1 or more. Lastly, subjects fulfilling neither normality nor dementia criteria were judged as cognitively impaired. This last group was divided into three different levels of impairment: memory, memory plus impairment of other cognitive functions, and memory plus impairment of other CDR categories.

At the end of the second diagnostic step, demented subjects were divided into different aetiological subgroups. To compare our findings with other studies, we collected data to rate the patients using the Hachinski ischaemic score ${ }^{26}$ (HIS), but our final diagnosis was made according to NINCDS-ADRDA ${ }^{27}$ and NINDS-AIREN ${ }^{28}$ criteria as follows: (1) probable Alzheimer's disease, when subjects had a progressive cognitive deterioration without systemic or brain disorders sufficient to produce dementia; (2) possible Alzheimer's disease, in the presence of clinical features not fulfilling the criteria of probable Alzheimer's disease, but without previous cerebrovascular events; (3) probable vascular dementia, when the onset of deterioration was abrupt and following (within three months) a recognised stroke that left focal neurological signs; (4) possible vascular dementia, in the presence of cerebrovascular events associated with clinical features not fulfilling the criteria of probable vascular dementia;(5) other dementing diseases, when a systemic or brain disorder was considered the cause of the dementia. The diagnosis was made according to the medical data, integrated with serum vitamin B12 determination and blood thyroid function tests when appropriate. As we were unable to perform MRI or CT on all demented patients, we used these only to exclude other causes of dementia.

The interrater reliability among the four investigators was tested after three months of training. The investigators independently examined the scheduled records of 40 subjects and made the diagnosis of both dementia and dementia subtype. The level of agreement was almost perfect $(k=0.83)$ for the diagnosis of dementia and substantial $(k=0.68)$ for the diagnosis of the five dementia subtypes.

DATA ANALYSIS AND STATISTICS

We calculated age specific and sex specific prevalence rates (cases per 100 population) for 
Table 1 Sociodemographic characteristics of study population*

\begin{tabular}{|c|c|c|c|c|c|c|}
\hline \multirow[b]{3}{*}{ Age (y): } & \multicolumn{2}{|l|}{ Men } & \multicolumn{2}{|c|}{ Women } & \multicolumn{2}{|c|}{ Total } \\
\hline & \multicolumn{2}{|c|}{ No $(\%)$} & \multicolumn{2}{|c|}{ No $(\%)$} & \multicolumn{2}{|c|}{ No $(\%)$} \\
\hline & & & & & & \\
\hline 65-69 & 117 & $(28 \cdot 0)$ & 151 & $(27 \cdot 5)$ & 268 & $(27 \cdot 7)$ \\
\hline $70-74$ & 126 & $(30 \cdot 1)$ & 150 & $(27 \cdot 3)$ & 276 & $(28 \cdot 5)$ \\
\hline $75-79$ & 89 & $(21 \cdot 3)$ & 119 & $(21 \cdot 6)$ & 208 & $(21 \cdot 5)$ \\
\hline $80-84$ & 62 & $(14 \cdot 8)$ & 89 & $(16 \cdot 2)$ & 151 & $(15 \cdot 6)$ \\
\hline $85-89$ & 17 & $(4 \cdot 1)$ & 25 & $(4 \cdot 5)$ & 42 & $(4 \cdot 3)$ \\
\hline $90-96 \dagger$ & 7 & $(1 \cdot 7)$ & 16 & $(2 \cdot 9)$ & 23 & $(2 \cdot 4)$ \\
\hline \multicolumn{7}{|l|}{ Education (y): } \\
\hline 0 & 16 & $(3 \cdot 8)$ & 16 & $(2 \cdot 9)$ & 32 & $(3 \cdot 3)$ \\
\hline $1-2$ & 61 & $(14 \cdot 6)$ & 146 & $(26 \cdot 5)$ & 207 & $(21 \cdot 4)$ \\
\hline $3-4$ & 113 & $(27 \cdot 0)$ & 249 & $(45 \cdot 3)$ & 362 & $(37 \cdot 4)$ \\
\hline 5 & 196 & $(46 \cdot 9)$ & 118 & $(21 \cdot 5)$ & 314 & $(32 \cdot 4)$ \\
\hline$>5$ & 32 & $(7 \cdot 7)$ & 21 & $(3 \cdot 8)$ & 53 & $(5 \cdot 5)$ \\
\hline \multicolumn{7}{|l|}{ Occupation: } \\
\hline Farmer & 107 & $(25 \cdot 6)$ & 318 & $(57 \cdot 8)$ & 425 & $(43 \cdot 9)$ \\
\hline Factory worker & 177 & $(42 \cdot 3)$ & 24 & $(4 \cdot 4)$ & 201 & $(20 \cdot 8)$ \\
\hline Housewife & - & - & 158 & $(28 \cdot 7)$ & 158 & $(16 \cdot 3)$ \\
\hline Other occupation & 134 & $(32 \cdot 1)$ & 50 & $(9 \cdot 1)$ & 184 & $(19 \cdot 0)$ \\
\hline Total for occupation $\ddagger$ & 418 & $(43 \cdot 2)$ & 550 & $(56 \cdot 8)$ & 968 & $(100 \cdot 0)$ \\
\hline
\end{tabular}

$\star$ Dropouts of phase $1(n=115)$ and phase $2(n=64)$ were excluded.

fOnly three subjects were over 94 .

In this line column (\%) refers to rows.

dementia of all types. Because of the small number of cases, prevalence rates for dementia subtypes were calculated by grouping together probable and possible cases and stratifying them in three age groups (10 year intervals) and two education groups (less than three years of schooling $v$ three or more years). The $95 \%$ confidence intervals (95\% CIs) of the proportions were calculated from the binomial distribution for $\mathbf{n}<100^{29}$ and the approximated formula based on the normal distribution in all the other cases. ${ }^{30}$

Univariate analysis was performed using $\chi^{2}$, Fisher's exact test, $\chi^{2}$ for trend, and MannWhitney $U$ test. Independent associations of putative risk factors with dementia were evaluated by means of stepwise logistic regression using $P<0.10$ as the selection threshold for significance. Analyses were processed using the 1990 release of the BMDP statistical software. $^{31}$

\section{Results}

Of the 1147 residents over 64 years of age at the prevalence day, $179(15 \cdot 6 \%)$ did not complete the protocol. Of the 115 subjects who dropped out during the first phase, 60 refused to be interviewed and 26 died before the interview could be conducted; 20 that had moved elsewhere were interviewed by phone and nine were irretrievably lost. Of the 64 subjects who dropped out during the second phase, three died before the clinical evaluation and 61 refused it. We were, therefore, able to obtain some information on $170(95 \%)$ of them. Dropouts and participants did not differ significantly with regard to sex $\left(\chi^{2}, \mathrm{P}=0.71\right)$, age (Mann-Whitney $U$ test, $P=0.66$ ), and education ( $18 \%$ with less than three years of schooling; $\chi^{2}, \mathrm{P}=0 \cdot 18$ ). Though none were institutionalised, $11(6.5 \%)$ were judged demented on the basis of information obtained from close informants and family doctors: three of the demented patients had less than three years of schooling and six were women.

Table 1 shows the sociodemographic characteristics of the 968 people who completed the protocol. Only two of them $(0 \cdot 2 \%)$ were institutionalised. Twenty one subjects with three or more years of schooling were screened by the MSQ because they had severe motor or sensory deficits. Of the 53 subjects with more than five years of schooling, only five were graduates. The proportion of subjects with a low level of education (less than three years of schooling) increased with age from $18.7 \%$ in the $65-69$ age group to $34.8 \%$ in the $90-96$ age group $\left(\chi^{2}\right.$ test for trend, $\left.P<0.001\right)$. The proportion of subjects with a low level of education was higher $(P<0.001)$ in women $(29.4 \%)$ than in men $(18.4 \%)$ and in farmers $(30.6 \%)$ than in subjects with other occupations $(13.0 \%)$. The mean age of farmers $(75.2$ (SD 6.6)) was higher $(P<0.01)$ than that of factory workers (73.7 (SD 6.0)) and of people with other occupations (73.4 (SD 6.5)).

Of the 968 subjects who completed the protocol (fig 1), 569 were judged as cognitively normal: $459(47 \cdot 4 \%, 95 \%$ CI $44 \cdot 3-50 \cdot 6)$ had negative screening tests; $110(11 \cdot 4 \%, 95 \%$ CI $9 \cdot 4-13 \cdot 4$ ) had positive screening tests but the clinical evaluation showed neither memory nor cognitive impairment.

Fifty seven subjects were judged as cognitively unclassifiable $(5 \cdot 9 \%, 95 \%$ CI $4 \cdot 4-7 \cdot 4)$. They had aphasia due to a previous stroke $(\mathrm{n}=$ $8)$, major depression ( $n=32)$, alcohol dependence $(n=9)$, schizophrenia $(n=4)$, severe personality disorders $(n=3)$, or mental retardation $(n=1)$. Eighteen patients with depression and four alcoholic patients had MMSE scores lower than 24 or MSQ scores lower than

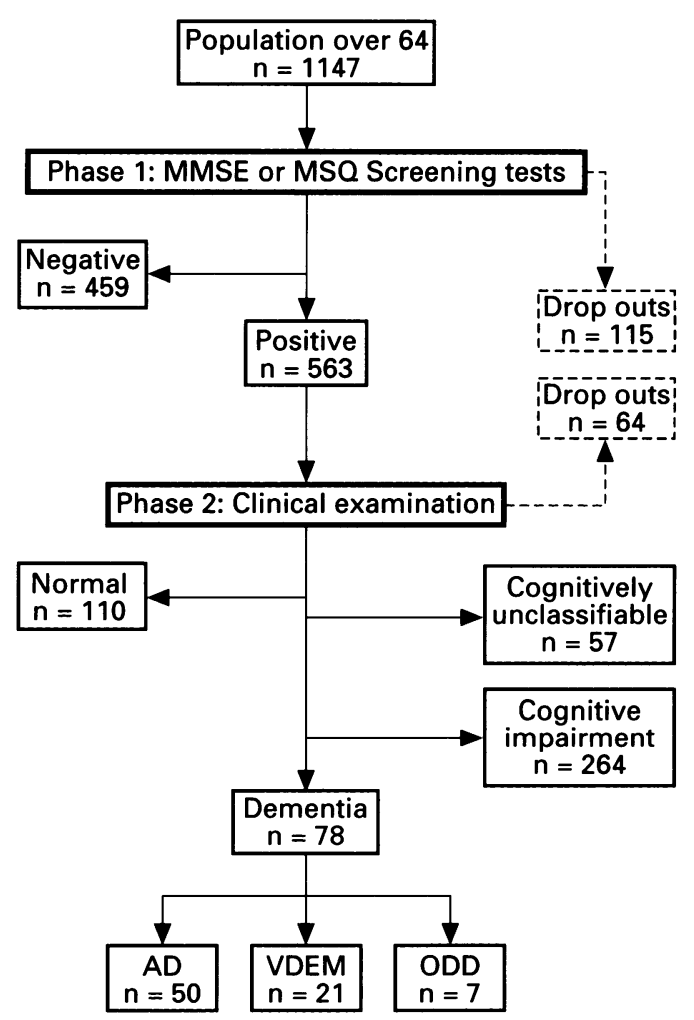

Figure 1 General design of the door to door two phase prevalence survey. The number of subjects involved in each step is shown. Subjects not traced, dead, or refusing to participate are indicated as dropouts. $A D=$ Alzheimer's disease; $V D E M=$ vascular dementia; $O D D=$ other dementing diseases. 
Table 2 Prevalance rates for dementia of all types by age and sex

\begin{tabular}{|c|c|c|c|c|c|c|}
\hline \multirow[b]{2}{*}{$\operatorname{Age}(y)$} & \multicolumn{2}{|c|}{ Men } & \multicolumn{2}{|c|}{ Women } & \multicolumn{2}{|c|}{ Total } \\
\hline & No & $P R(95 \% C I)$ & No & $P R(95 \% C I)$ & No & $P R(95 \% C I)$ \\
\hline $\begin{array}{l}65-69 \\
70-74 \\
75-79 \\
80-84 \\
85-89 \\
90-96\end{array}$ & $\begin{array}{r}1 \\
2 \\
9 \\
14 \\
5 \\
2\end{array}$ & $\begin{array}{c}0.9(0 \cdot 0-2 \cdot 5) \\
1 \cdot 6(0 \cdot 0-3 \cdot 8) \\
10 \cdot 1(4 \cdot 7-18 \cdot 3) \\
22 \cdot 6(12 \cdot 9-35 \cdot 0) \\
29 \cdot 4(10 \cdot 3-56 \cdot 0) \\
28 \cdot 6(3 \cdot 7-71 \cdot 0)\end{array}$ & $\begin{array}{r}2 \\
7 \\
5 \\
20 \\
5 \\
6\end{array}$ & $\begin{array}{c}1 \cdot 3(0 \cdot 0-3 \cdot 1) \\
4 \cdot 7(1 \cdot 3-8 \cdot 0) \\
4 \cdot 2(0 \cdot 6-7 \cdot 8) \\
22 \cdot 5(14 \cdot 3-32 \cdot 6) \\
20 \cdot 0(6 \cdot 8-40 \cdot 7) \\
37 \cdot 5(15 \cdot 2-64 \cdot 6)\end{array}$ & $\begin{array}{r}3 \\
9 \\
14 \\
34 \\
10 \\
8\end{array}$ & $\begin{array}{c}1 \cdot 1(0 \cdot 0-2 \cdot 4) \\
3 \cdot 3(1 \cdot 2-5 \cdot 3) \\
6 \cdot 7(3 \cdot 3-10 \cdot 1) \\
22 \cdot 5(15 \cdot 9-29 \cdot 2) \\
23 \cdot 8(12 \cdot 1-39 \cdot 5) \\
34 \cdot 8(16 \cdot 4-57 \cdot 3)\end{array}$ \\
\hline Total & 33 & $7.9(5 \cdot 3-10 \cdot 5)$ & 45 & $8 \cdot 2(5.9-10 \cdot 5)$ & 78 & $8 \cdot 0(6 \cdot 3-9 \cdot 8)$ \\
\hline
\end{tabular}

$\mathrm{PR}=$ Prevalence rate (cases per 100 population).

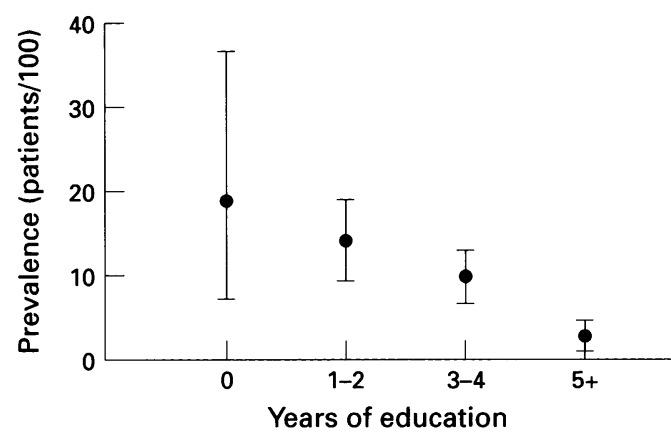

Figure 2 Association of years of schooling with dementia expressed as prevalence ( $95 \%$ confidence intervals).

Table 3 Prevalence rates for dementia of all types by occupation and education

\begin{tabular}{lrc}
\hline & $N o$ & $P R(95 \% C I)$ \\
\hline Occupation: & 39 & $9 \cdot 2(6 \cdot 4-11 \cdot 9)$ \\
$\quad$ Farmer & 21 & $10 \cdot 4(6 \cdot 2-14 \cdot 7)$ \\
Factory worker & 8 & $5 \cdot 1(1 \cdot 6-8 \cdot 5)$ \\
Housewife & 10 & $5 \cdot 4(2 \cdot 2-8 \cdot 7)$ \\
Other occupation & & $18 \cdot 7(7 \cdot 2-36 \cdot 4)$ \\
Education (y): & 6 & $14 \cdot 0(9 \cdot 3-18 \cdot 7)$ \\
0 & 29 & $9 \cdot 7(6 \cdot 6-12 \cdot 7)$ \\
$1-2$ & 35 & $2 \cdot 5(0 \cdot 8-4 \cdot 3)$ \\
$3-4$ & 8 & $0 \cdot 0(0 \cdot 0-6 \cdot 7)$ \\
5 & 0 &
\end{tabular}

8 but their cognitive impairment was not sufficiently severe to warrant a diagnosis of dementia.

Of the 264 subjects with cognitive impairment $(27 \cdot 3 \%, 95 \%$ CI $24 \cdot 5-30 \cdot 1), 149$ $(56.4 \%)$ had CDR memory scores of 0.5 or 1 , but no other cognitive disturbances; 53 $(20 \cdot 1 \%)$ had memory impairment and other cognitive disturbances, but they scored 0 in the other CDR categories. The remaining 62 subjects $(23.5 \%)$ had memory impairment and abnormal scores in other CDR categories; 31 of these were fully assessable and their global CDR score was 0.5 ; the remaining 31 had physical problems or daily life activities that hampered the correct evaluation of one or more CDR categories: 17 of them scored 1 in

Table 4 Prevalence rates for dementia of all types by age, sex, and education

\begin{tabular}{|c|c|c|c|c|c|}
\hline & \multicolumn{2}{|c|}{$<3$ years of schooling } & \multicolumn{2}{|c|}{$\geqslant 3$ years of schooling } & \multirow[b]{2}{*}{ Pvalue* } \\
\hline & No & $P R(95 \% C I)$ & No & $P R(95 \% C I)$ & \\
\hline \multicolumn{6}{|l|}{ Sex: } \\
\hline $\begin{array}{l}\text { Men } \\
\text { Women }\end{array}$ & $\begin{array}{l}14 \\
21\end{array}$ & $\begin{array}{l}18 \cdot 2(10 \cdot 3-28 \cdot 6) \\
13 \cdot 0(7 \cdot 8-18 \cdot 1)\end{array}$ & $\begin{array}{l}19 \\
24\end{array}$ & $\begin{array}{l}5 \cdot 6(3 \cdot 1-8 \cdot 0) \\
6 \cdot 2(3 \cdot 8-8 \cdot 6)\end{array}$ & $\begin{array}{r}<0.001 \\
0.013\end{array}$ \\
\hline $\begin{array}{l}\text { Women } \\
\text { Age (y): }\end{array}$ & & & & & \\
\hline $\begin{array}{l}65-74 \\
75-84 \\
85-96\end{array}$ & $\begin{array}{r}6 \\
21 \\
8\end{array}$ & $\begin{array}{c}5 \cdot 8(1 \cdot 3-10 \cdot 2) \\
19 \cdot 6(12 \cdot 1-27 \cdot 2) \\
28 \cdot 6(11 \cdot 8-45 \cdot 3)\end{array}$ & $\begin{array}{r}6 \\
27 \\
10\end{array}$ & $\begin{array}{l}1 \cdot 4(0 \cdot 3-2 \cdot 4) \\
10 \cdot 7(6 \cdot 9-14 \cdot 5) \\
27 \cdot 0(12 \cdot 7-41 \cdot 3)\end{array}$ & $\begin{array}{l}0.017 \\
0.036 \\
0.887\end{array}$ \\
\hline Total & 35 & $14 \cdot 6(10 \cdot 2-19 \cdot 1)$ & 43 & $5 \cdot 9(4 \cdot 2-7 \cdot 7)$ & $<0.001$ \\
\hline
\end{tabular}

$\mathrm{PR}=$ Prevalence rate (cases per 100 population).

${ }^{\star} \chi^{2}$ test.
Table 5 Prevalence rates for Alzheimer's disease and vascular dementia according to age, sex, and education

\begin{tabular}{|c|c|c|c|c|}
\hline & \multicolumn{2}{|c|}{ Alzheimer's disease } & \multicolumn{2}{|c|}{ Vascular dementia } \\
\hline & No & $P R(95 \% C I)$ & No & $P R(95 \% C I)$ \\
\hline $\begin{array}{r}\text { Age }(y): \\
65-74 \\
75-84 \\
85-96\end{array}$ & $\begin{array}{r}7 \\
32 \\
11\end{array}$ & $\begin{array}{c}1 \cdot 3(0 \cdot 4-2 \cdot 2) \\
8 \cdot 9(6 \cdot 0-11 \cdot 9) \\
16 \cdot 9(8 \cdot 8-28 \cdot 3)\end{array}$ & $\begin{array}{r}3 \\
12 \\
6\end{array}$ & $\begin{array}{l}0 \cdot 6(0 \cdot 0-1 \cdot 2) \\
3 \cdot 3(1 \cdot 5-5 \cdot 2) \\
9 \cdot 2(3 \cdot 5-19 \cdot 0)\end{array}$ \\
\hline $\begin{array}{l}\text { Sex: } \\
\text { Men } \\
\text { Women }\end{array}$ & $\begin{array}{l}21 \\
27\end{array}$ & $\begin{array}{l}5 \cdot 0(2 \cdot 9-7 \cdot 1) \\
4 \cdot 9(3 \cdot 1-6 \cdot 7)\end{array}$ & $\begin{array}{r}9 \\
12\end{array}$ & $\begin{array}{l}2 \cdot 2(0 \cdot 8-3 \cdot 5) \\
2 \cdot 2(1 \cdot 0-3 \cdot 4)\end{array}$ \\
\hline $\begin{array}{l}\text { Education }(y) \text { : } \\
\quad<3 \\
\quad \geqslant 3\end{array}$ & $\begin{array}{l}22 \\
28\end{array}$ & $\begin{array}{l}9 \cdot 2(5 \cdot 5-12 \cdot 9) \\
3 \cdot 8(2 \cdot 4-5 \cdot 2)\end{array}$ & $\begin{array}{l}10 \\
11\end{array}$ & $\begin{array}{l}4 \cdot 2(1 \cdot 6-6 \cdot 7) \\
1 \cdot 5(0 \cdot 6-2 \cdot 4)\end{array}$ \\
\hline Total & 50 & $5 \cdot 2(3 \cdot 8-6 \cdot 6)$ & 21 & $2 \cdot 2(1 \cdot 3-3 \cdot 1)$ \\
\hline
\end{tabular}

$\mathrm{PR}=$ Prevalence rate (cases per 100 population).

memory and 0.5 in judgement or orientation, 13 scored 1 in memory and judgement, and one scored 1 in memory and orientation.

Dementia was diagnosed in 78 patients $(8 \cdot 0 \%, 95 \%$ CI $6 \cdot 4-9 \cdot 8)$. None of them had MMSE scores over 23 or MSQ scores over 8 . Dementia was judged as mild in 36 patients (3.7\% of participants), moderate in $29(3.0 \%)$, and severe in $13(1.3 \%)$. The overall prevalence of dementia did not differ between men and women $(P=0.97)$, but increased with age in both sexes, roughly doubling every five years of age. Prevalence rates for dementia were higher in farmers $(9 \cdot 2 \%, 95 \%$ CI $6 \cdot 4-11.9)$ and in factory workers $(10.4 \%, 95 \%$ CI $6 \cdot 2-14 \cdot 7)$ than in housewives $(5 \cdot 1 \%, 95 \%$ CI $1 \cdot 6-8 \cdot 5)$ and subjects with other occupations $(5 \cdot 4 \%, 95 \%$ CI $2 \cdot 2-8 \cdot 7)$, but these differences were not statistically significant $(P=0 \cdot 12)$ (table 2).

Figure 2 shows that prevalence of dementia and years of education were inversely related with a highly significant dose-effect relation $\left(\chi^{2}\right.$ for trend: $\mathrm{P}<0.0001)$. When age specific and sex specific prevalence rates of dementia were stratified in two education levels, the effect of education was similar in both sexes and tended to disappear with advancing age (table 3). At the logistic analysis, the OR for dementia of subjects with a low level of education was $2 \cdot 7$ (95\% CI 1.7-4.5) and was still significant when age and sex were added to the model (adjusted OR $2 \cdot 0 ; 95 \%$ CI $1 \cdot 2-3 \cdot 3$ ). The proportion of low educated patients in mild (43\%) and moderate-severe (48\%) demented groups did not differ significantly $\left(\chi^{2}, \mathrm{P}=0.76\right)$.

Of the 78 demented subjects, $50(64 \%)$ were diagnosed as having Alzheimer's disease (37 probable and 13 possible cases) and 21 $(27 \%)$ as having vascular dementia (11 probable and 10 possible cases). The remaining seven patients (9\%), included in the other dementing diseases category, had Parkinson's disease $(n=4)$, severe cardiorespiratory disease $(n=2)$, or chronic renal failure $(n=1)$. Of the 50 patients with Alzheimer's disease, 35 had HIS scores under 5 , and 15 (eight probable and seven possible) had HIS scores of 5 or 6 . Of the 21 patients with vascular dementia, 18 had HIS scores over 6 and three possible cases had HIS scores of 5 or 6 .

Table 4 shows the age, sex, and education specific prevalence rates for Alzheimer's disease and vascular dementia. In both dementia subtypes, the prevalence rates did not differ 
between men and women, but increased with age and were higher in subjects with a low level of education. The age adjusted OR of subjects with a low level of education was $1 \cdot 8$ (95\% CI 1.0-3.4) for Alzheimer's disease and $2 \cdot 1(95 \%$ CI $0 \cdot 9-5 \cdot 3)$ for vascular dementia.

\section{Discussion}

Differences in diagnostic criteria and case ascertainment procedures can produce pronounced variations in prevalence estimates of dementia. In the present study the overall prevalence for dementia, calculated on the 968 subjects who completed the protocol, was $8 \cdot 0$. As we obtained clinical information on $95 \%$ of dropouts and 11 of them were judged demented, the prevalence would have been marginally lower $(7 \cdot 8)$ if we had calculated it on all the 1147 residents. The risk of missing demented patients before the prevalence day was minimal, given that only two of the eligible subjects had moved elsewhere during the five years preceding the study. On the other hand, if we had considered the 31 subjects with CDR memory scores of 1 and missing scores in other CDR categories as demented, the prevalence of dementia would have been $11 \cdot 3(109 / 968)$. These data confirm that even small changes in diagnostic criteria can produce differences in prevalence estimates which are much more remarkable than those caused by bias in case ascertainment procedures. However, to compare our data with other population studies we have to consider the effects of other variables.

We found, as have other studies, ${ }^{12}$ that the prevalence of dementia increased with age, roughly doubling every five years. Although some studies ${ }^{6932}$ have found higher prevalence rates for dementia in women than in men, ours, in agreement with others, ${ }^{35}$ did not find any relation between sex and risk of dementia. This discrepancy could be explained by the effect of other factors such as education, occupation, or socioeconomic state.

According to recent studies, ${ }^{9-13}$ illiterate or poorly educated subjects had a two to three times greater risk of dementia than highly educated subjects. Despite the low educational level of our population (only $5.5 \%$ with more than five years of schooling), we found a similar association between education and dementia. We do not think this effect stems from a bias in either screening or diagnostic procedures. It is unlikely that we missed cases of dementia among subjects with more than three years of schooling because we used an MMSE cut off point higher than that usually employed in populations with a better level of education. Moreover, we can exclude a selection bias because the proportion of low educated subjects did not differ significantly between dropouts and participants, and three of the 11 demented dropouts had less than three years of schooling. Lastly, to prevent spurious diagnoses of dementia in low educated people, we used diagnostic criteria requiring formal evidence of functional impairment: the lack of an increased proportion of low educated subjects in the group of mild demented patients speaks against this kind of misdiagnosis.

Old age and female sex were significantly associated with low education, but we found that the association between education and dementia was still present after adjusting for these potential confounders $(O R=2 \cdot 0 ; 95 \%$ CI 1-1-3:1). By contrast with the Shanghai study, ${ }^{9}$ we did not find any relation between female sex and a low level of education, and risk of dementia. Zhang $e t a l^{9}$ attributed such a correlation in this study to the higher mortality in men with dementia; this bias is unlikely to be present in our population because the prevalence of dementia did not differ between men and women.

Some studies ${ }^{133}$ have found a significant correlation between occupation and dementia, although this effect has not been confirmed by others. ${ }^{34}$ Because education, occupation, and socioeconomic state are often associated, it is difficult to determine the effect of each variable. As most subjects in our study population had manual occupations, we were unable to explore the possible effects of non-manual occupations, but we can reasonably exclude occupation as a confounder of education given that we did not find a significant association between occupation and dementia. Moreover, the potential confounding effects of cultural activities and socioeconomic state were negligible because these variables did not differ substantially in subjects with different occupations or educational levels. Lastly, although low education is a normal connotation of this rural population, we cannot exclude the possibility that a very low educational attainment was associated with low cognitive abilities or other personal characteristics likely to increase the risk of dementia. However, as the threshold of three years of education is closely related to literacy, the increased risk of dementia might be better explained by literacy than by minimal differences in years of schooling.

The proportion of cases of Alzheimer's disease (probable and possible cases grouped together) was $64 \%$, a figure similar to the Shanghai ${ }^{9}(65 \%)$ and Rochester ${ }^{3}(67 \%)$ studies, but slightly lower than that in the Rotterdam study ${ }^{10}(72 \%)$. However, if we had considered all the patients with HIS scores over 4 as cases of vascular dementia, the proportion of those with vascular dementia would have increased from $27 \%$ to $46 \%$, a figure closer to the one found in other studies that used this cut off point to differentiate Alzheimer's disease from vascular dementia. ${ }^{735}$

In agreement with other studies, ${ }^{35}$ prevalence rates did not differ between men and women and increased with age in both Alzheimer's disease and vascular dementia groups. Owing to the limited number of cases, we cannot tell whether women had a higher risk of Alzheimer's disease in the most advanced ages, as has been reported by others. ${ }^{7}$ Lastly, we found, as did the Rotterdam study, ${ }^{10}$ that a low level of education increased the risk of dementia in both dementia subtypes but, owing to the few cases, the lower confidence limits of adjusted ORs was 1.0 for 
Alzheimer's disease and 0.9 for vascular dementia. Therefore, the protective effect of education may operate by mechanisms irrespective of pathogenic determinants.

In conclusion, our data suggest that prevalence of dementia both increases with age and is inversely related to education, even in this population characterised by a limited degree of education and no substantial differences in lifestyle. The effects of age and education are similar in both sexes and in both dementia subtypes.

We thank Dr Paola Calisse and the lay interviewers ML We thank Dr Paola Calisse and the lay interviewers ML their data collection. We are grateful to the family doctors and their data collection. We are grateful to the fam

the subjects themselves for their cooperation.
This work was supported in part by Grants from Pierrel Spa This work was supported in part by Grants from Pierrel Spa
and National Research Council (CNR-No 94.00471.PF40).

1 Jorm AF, Korten AE, Henderson AS. The prevalence of dementia: a quantitative integration of literature. Acta Psychiatr Scand 1987;76:465-79.

2 Hofman A, Rocca WA, Brayne C, et al. The prevalence of dementia in Europe: a collaborative study of 1980-1990 findings. Int $\mathcal{F}$ Epidemiol 1991;20:736-48.

3 Kokmen E, Beard CM, Offord KP, Kurland LT. Prevalence of medically diagnosed dementia in a defined United States population: Rochester, MN, January 1, United States population: Roch
1975. Neurology 1989;39:773-6.

4 Evans DA, Funkenstein $\mathrm{HH}$, Albert MS, et al. Prevalence of Alzheimer's disease in a community population of of Alzheimer's disease in a community population of older persons. Hi
$1989 ; 262: 2551-6$.

5 Fratiglioni L, Grut M, Forsell Y, Viitanen M, et al. Prevalence of Alzheimer's disease and other dementias in an elderly urban population: relationship with age, sex and education. Neurology 1991;41:1886-92.

6 Bachman DL, Wolf PA, Linn R, et al. Prevalence of dementia and probable senile dementia of the Alzheime type in the Framingham Study. Neurology 1992;42. 115-9.

7 Rocca WA, Bonaiuto S, Lippi A, et al. Prevalence of clinically diagnosed Alzheimer's disease and other dementing disorders: a door-to-door survey in Appignano, Macerata disorders: a door-to-door survey in Appignano

8 Brayne C, Calloway P. An epidemiological study of dementia in a rural population of elderly women. Br $\mathcal{F}$ Psychiatry 1989;155:214-9.

9 Zhang M, Katzman R, Salmon D, et al. The prevalence of dementia and Alzheimer's disease in Shanghai, China: impact of age, gender, and education. Ann Neurol 1990; 27:428-37

10 Ott A, Breteler MMB, van Harskamp F, et al. Prevalence of Alzheimer's disease and vascular dementia: association with education. The Rotterdam study. $B M F$ 1995;310: 970-3.

11 Mortimer JA, Graves AB. Education and other socioeconomic determinants of dementia and Alzheimer's disease. Neurology 1993;43(suppl 4):S39-44.

12 Katzman R. Education and the prevalence of dementia and Alzheimer's disease. Neurology 1993;43:13-20.
13 Stern Y, Gurland B, Tatemichi TK, Tang MX, Wilder D, Mayeux R. Influence of education and occupation on the incidence of Alzheimer's disease. $\mathcal{F A M A} 1994 ; 271$ : 1004-10.

14 Folstein MF, Folstein SE, McHugh PR. Mini-mental state: a practical method for grading the cognitive state of a practical method for grading the cognitive state of

15 Kahn RL, Goldfarb AI, Pollack M, Peck A. Brief objective measures for the determination of mental status in the aged. Am F Psychiatry 1960;117:326-8.

16 Cohen J. A coefficient of agreement for nominal scales. Educ Psychol Meas 1960;20:37-46.

17 Landis JR, Koch CG. The measurement of observer agreement for categorical data. Biometrics 1977;33:159-74

18 Hamilton $M$. Development of a rating scale for primary depressive illness. Br $\mathcal{f}$ Soc Clin Psychology 1967;6: 278-96.

19 Huges CP, Berg L, Danziger WL, Coben LA, Martin RL. A new clinical scale for the staging of dementia. $\mathrm{Br} \mathcal{F}$ Psychiatry 1982;140:566-72

20 Broe GA, Akhtar AJ, Andrews GR, et al. Neurological disorders in the elderly at home. $\mathcal{f}$ Neurol Neurosurg Psychiatry 1976;39:362-6.

21 Kaplan E, Goodglass $\mathrm{H}$, Weintraub S. The Boston naming test. Philadelphia: Lea and Febiger, 1983

22 De Renzi E, Motti F, Nichelli P. Imitating gestures. A quantitative approach to ideomotor apraxia. Arch Neurol 1980;37:6-10.

23 Sunderland T, Hill JL, Mellow AM, et al. Clock drawing in Alzheimer's disease: a novel measure of dementia severity. F Am Geriatr Soc 1989;37:725-9

24 American Psychiatric Association. Diagnostic and statistical manual of mental disorders, 3rd ed, revised. Washington, DC: American Psychiatric Association, 1987.

25 Morris JC. The clinical dementia rating (CDR): current version and scoring rules. Neurology 1993;43:2412-4.

26 Hachinski VC, Iliff LD, Zilhka E, et al. Cerebral blood flow in dementia. Arch Neurol 1975;32:632-7.

27 McKhann G, Drachman D, Folstein M, Katzman R, Price D, Stadlan EM. Clinical diagnosis of Alzheimer's disease: report of the NINCDS-ADRDA Work Group under the auspices of Department of Health and Human Services Task Force on Alzheimer's Disease. Neurology 1984;34:939-44.

28 Romàn GC, Tatemichi TK, Erkinjuntti T, et al. Vascular dementia: diagnostic criteria for research studies. Report of the NINDS-AIREN International Workshop. Neurology 1993;43:250-60.

29 Lentner C, ed. Tavole Scientifiche Geigy. 8th Italian ed. Basel: CIBA-GEIGY Limited, 1983:2;89-102.

30 Armitage P, Berry G. Statistical methods in medical research. 2nd ed. Oxford: Blackwell, 1987:115-20.

31 Dixon WJ, Chief ed. BMDP Statistical software, 1990 release. Berkeley: University of California Press, 1990

32 Coria F, Gomez de Caso JA, Minguez L, RodriguezCoria F, Gomez de Caso JA, Minguez L, Rodriguez-
Artalejo F, Claveria LE. Prevalence of age-associated memory impairment and dementia in a rural community. f Neurol Neurosurg Psychiatry 1993;56:973-6.

33 Bonaiuto S, Rocca WA, Lippi A, et al. Education and occupation as risk factors for dementia: a population-based case-control study. Neuroepidemiology 1995;14:101-9.

34 Beard CM, Kokmen E, Offord KP, Kurlaand LT. Lack of association between Alzheimer's disease and education, occupation, marital status, or living arrangement. Neurology 1992;42:2063-8.

35 Skoog I, Nilsson L, Palmertz B, Andreasson LA, Svanborg A. A population-based study of dementia in 85 -year-olds. N Engl $\mathcal{F}$ Med 1993;328:153-8. 